\title{
Cobalt-60 gamma irradiation of silicon test structures for high-luminosity collider experiments
}

\author{
Patrick Asenov $^{\star a}$, Panagiotis Assiouras ${ }^{a}$, Argiro Boziari $^{b}$, Konstantinos Filippou $^{c}$, \\ Ioannis Kazas $^{a}$, Aristotelis Kyriakis ${ }^{a}$ and Dimitrios Loukas ${ }^{a}$ \\ *E-mail: patrick. asenov. asenovacern. ch \\ ${ }^{a}$ Institute of Nuclear and Particle Physics (INPP), NCSR Demokritos, \\ Aghia Paraskevi, Greece \\ ${ }^{b}$ Greek Atomic Energy Commission (GAEC), \\ Aghia Paraskevi, Greece \\ ${ }^{c}$ University of Ioánnina, \\ Ioánnina, Greece
}

\begin{abstract}
During the era of the High Luminosity LHC (HL-LHC) the devices in the experiments will be subjected to increased radiation levels with fluxes of neutrons and charged hadrons in the inner detectors reaching up to approximately $2.3 \times 10^{16} \mathrm{n}_{\mathrm{eq}} / \mathrm{cm}^{2}$ and total ionization doses up to around $1.2 \mathrm{Grad}$. A systematic program of radiation tests with neutrons and charged hadrons is being carried out by the CMS and ATLAS Collaborations in order to cope with the higher luminosity of HL-LHC and the associated increase in the pile-up events and radiation fluxes. In this work, results from a complementary radiation study with ${ }^{60} \mathrm{Co}-\gamma$ photons are presented. The doses are equivalent to those that the outer layers of the silicon tracker systems of the two experiments will be subjected to. The devices in this study are p-type diodes and MOS capacitors.
\end{abstract}

The 28th International Workshop on Vertex Detectors - Vertex2019

13-18 October, 2019

Lopud, Croatia

${ }^{*}$ Speaker. 


\section{Introduction}

In the coming years, the Large Hadron Collider (LHC) is getting upgraded to the High Luminosity LHC (HL-LHC) with the scope of boosting the accelerator's potential for new discoveries in physics and studying rare processes. The aim is to increase the luminosity of the collider by a factor of 10 , up to $10^{35} \mathrm{~cm}^{-2} \mathrm{~s}^{-1}$, with the integrated luminosity expected to reach $3000 \mathrm{fb}^{-1}$ [1]. For this reason, new silicon tracking detectors with improved radiation hardness are required for the HLLHC experiments. In the HL-LHC era the total absorbed doses in the outer layers of the tracking systems are estimated to be in the order of 10-100 kGy, depending on the distance from the beam line [2], [3]. A systematic campaign of irradiation tests with neutrons and charged hadrons initiated by the LHC collaborations is ongoing in order to estimate how will the candidate devices for the tracking systems cope with a) the higher luminosity of the HL-LHC and b) the associated increase in pile-up events and radiation fluxes. Complementary radiation studies with ${ }^{60} \mathrm{Co}-\gamma$ photons are performed with the doses equivalent to those that the outer layers of the silicon tracker systems of the two large LHC experiments will be subjected to. One of the studies is presented here.

\section{Samples and laboratory equipment}

The samples used for irradiation in this study are float-zone oxygenated silicon n-in-p test structures from thinned $240 \mu \mathrm{m}$ thick wafers produced by Hamamatsu Photonics K.K. [4]. Each test structure contains one square MOS (area $=4 \mathrm{~mm} \times 4 \mathrm{~mm}$ ) and two square diodes (area $=2.5$ $\mathrm{mm} \times 2.5 \mathrm{~mm}$ and area $=1.25 \mathrm{~mm} \times 1.25 \mathrm{~mm}$, respectively).

The ${ }^{60} \mathrm{Co}$ source is a Picker teletherapy unit [5] with a radioactivity of $30 \mathrm{TBq}$ as of March 2012, estimated at approximately $11 \mathrm{TBq}$ today, with a horizontal orientation (Fig. 1, left). It was calculated using FC65-P Ionization Chambers from IBA Dosimetry [6] that the dose rate at irradiation point ( $40 \mathrm{~cm}$ from the source) is $\sim 1 \mathrm{kGy} / \mathrm{h}$. The irradiation was performed in the secondary standard ionizing radiation laboratory of the Greek Atomic Energy Commission (GAEC), accredited according to ISO 17025 in the field of radiotherapy, and the relevant CMCs (calibration and measurement capabilities) are published in the BIPM database [7]. The cooling system consisted of a thermoelectric cooler (Peltier element, type TEC1 12704) operating at $20^{\circ} \mathrm{C}$, an aluminum plate and a fan for heat dissipation (Fig. 1, right). The selected Peltier is sealed with 704 silicon rubbers and proved to be robust against $\gamma$-irradiation from the Cobalt-60 source. A microcontroller for the stabilization of temperature and the respective power supplies were used in addition (Fig. 2, left). Charged particle equilibrium (CPE) was achieved due to a box of $2 \mathrm{~mm}$ thick $\mathrm{Pb}$ and $0.8 \mathrm{~mm}$ of inner lining $\mathrm{Al}$ sheet, i.e. a lead-aluminum container for the absorption of low energy photons and secondary electrons [8] where the samples were kept during irradiation (Fig. 2, right).

Electrical measurements were performed using an automatic probe station (Carl Suss PA 150) and supplementary equipment (HP4092A, Keithley 6517A) for electrical characterization of microelectronic devices and the samples were annealed in a Weiss WKS 3-180/40/5 climate test chamber.

\section{Experimental procedure and protocol}

The irradiation was split in slots of 5 hours of irradiation. After each slot, annealing of the 

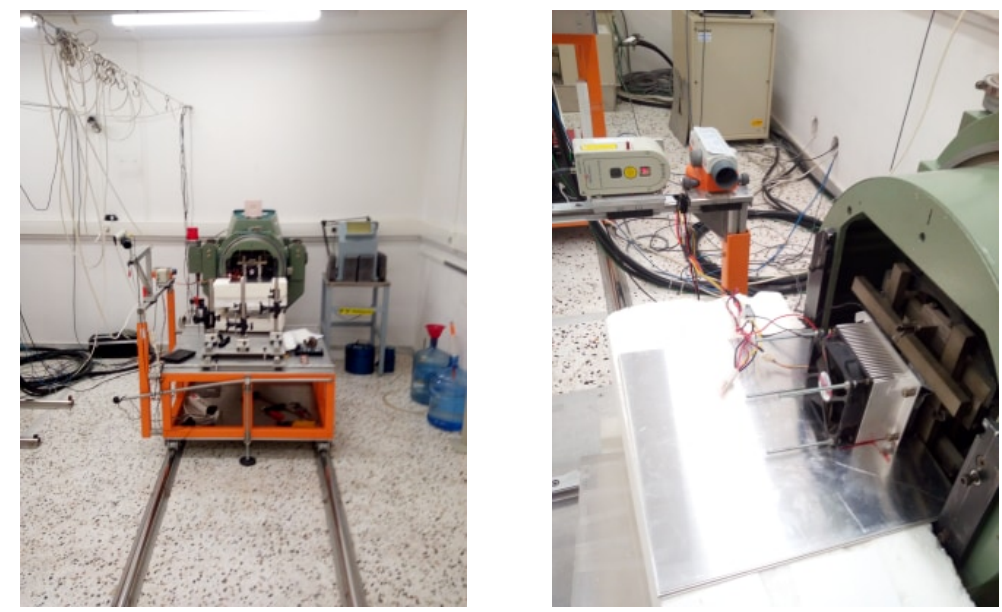

Figure 1: Left: The Cobalt-60 source: Picker therapy unit. Right: The container with the samples in front of the source. The fan and the thermoelectric cooler are visible.
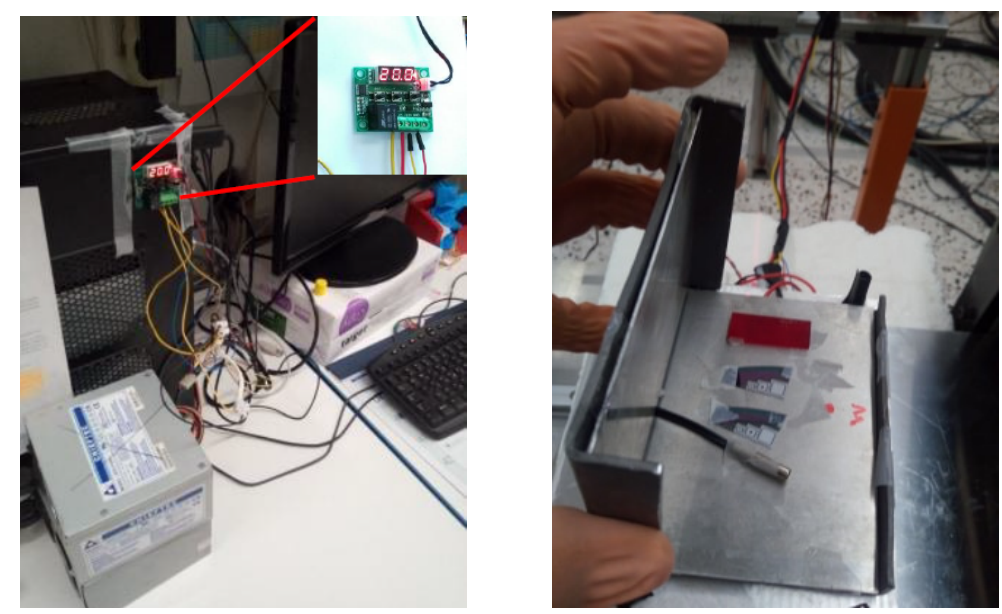

Figure 2: Left: The microcontroller and power supplies of the experimental setup. Right: The leadaluminum container for charged particle equilibrium. Two samples can also be seen here (gray-colored test structures). Each of the two structures contains (from left to right): square diode with area $=1.25 \mathrm{~mm} \times$ $1.25 \mathrm{~mm}$, square diode with area $=2.5 \mathrm{~mm} \times 2.5 \mathrm{~mm}$, square MOS capacitor with area $=4 \mathrm{~mm} \times 4 \mathrm{~mm}$.

samples was performed in the climate test chamber at $60{ }^{\circ} \mathrm{C}$ for $10 \mathrm{~min}$ (corresponding to four days of annealing at room temperature). The electrical tests at the probe station after the annealing were performed at $22-24{ }^{\circ} \mathrm{C}$ using LabVIEW as operation and control software. The oscillation amplitude for the $\mathrm{CV}$ measurements was set at $250 \mathrm{mV}$. CV measurements were carried out for various frequencies $(100 \mathrm{~Hz}, 1 \mathrm{kHz}, 10 \mathrm{kHz}, 100 \mathrm{kHz}, 1 \mathrm{MHz}$ for MOS capacitors; $10 \mathrm{kHz}, 100$ $\mathrm{kHz}, 1 \mathrm{MHz}$ for diodes). Between each set of electrical measurements and the following irradiation slot, the samples were stored in a freezer at $-28{ }^{\circ} \mathrm{C}$.

\section{Results from MOS (CV analysis)}

After the exposure to gamma photons there is a clear evidence of positive charge induced in 


\begin{tabular}{|c|c|c|c|c|c|}
\hline Absorbed dose $[\mathrm{kGy}]$ & $\mathrm{C}_{\mathrm{acc}}[\mathrm{pF}]$ & $\mathrm{C}_{\mathrm{inv}}[\mathrm{pF}]$ & $\mathrm{t}_{\mathrm{Ox}}[\mu \mathrm{m}]$ & $\mathrm{V}_{\mathrm{FB}}[\mathrm{V}]$ & $\mathrm{N}_{\mathrm{a}}\left[\mathrm{cm}^{-3}\right]$ \\
\hline 0 & 851.65 & 203.42 & 0.65 & -1.32 & $8.30 \times 10^{12}$ \\
\hline 5 & 839.06 & 202.75 & 0.66 & -22.80 & $8.37 \times 10^{13}$ \\
\hline 10 & 835.72 & 202.75 & 0.66 & -29.80 & $8.16 \times 10^{13}$ \\
\hline 15 & 835.73 & 187.41 & 0.66 & -36.43 & $9.12 \times 10^{13}$ \\
\hline 25 & 811.08 & 178.22 & 0.68 & -43.66 & $1.01 \times 10^{14}$ \\
\hline 30 & 803.90 & 182.97 & 0.69 & -45.71 & $1.03 \times 10^{14}$ \\
\hline
\end{tabular}

Table 1: Various features of a MOS capacitor before and after irradiation; measurement frequency $=10$ kHz. (Derived from Chapter 6 of [9].)

the oxide of the p-type MOS capacitors as seen in Fig. 3, where a shift of the flat band voltage $\left(\mathrm{V}_{\mathrm{FB}}\right)$ to higher absolute values is observed at higher doses. This also implies an increase of the doping concentration $\left(\mathrm{N}_{\mathrm{a}}\right)$. Some other features of MOS capacitors (capacitance in the accumulation region $\mathrm{C}_{\mathrm{acc}}$, capacitance in the inversion region $\mathrm{C}_{\mathrm{inv}}$, oxide thickness $\mathrm{t}_{\mathrm{ox}}$ ) before and after irradiation are summarized in Table 1. The derivation for these have been done by known rules [9]. In the analysis it is noticed that the oxide thickness remains stable as expected, since it is a geometric characteristic of the device. In addition, with higher doses the transition from the depletion region to the inversion region becomes inertial due to the delay of the generation of carriers while increasing the voltage.

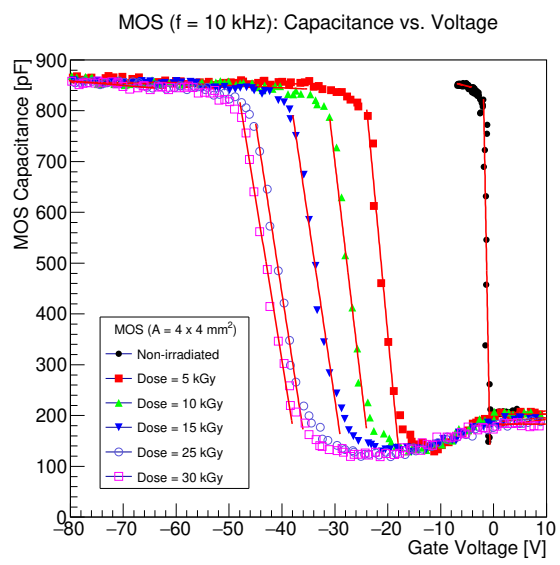

Figure 3: Capacitance-voltage curve for a MOS capacitor for various doses; measurement frequency $=10$ $\mathrm{kHz}$.

\section{Results from a $2.5 \mathrm{~mm}$ sized diode (CV and IV analysis)}

The CV curves of a $2.5 \mathrm{~mm}$ sized diode before and after irradiation are almost identical (Fig. 4, left). Using $1 / \mathrm{C}^{2}$ calculated after the $\mathrm{CV}$ measurement (Fig. 4, right), useful information about the depletion voltage $\left(\mathrm{V}_{\mathrm{d}}\right)$, capacitance in the depletion region $\left(\mathrm{C}_{\mathrm{d}}\right)$ and acceptor concentration $\left(\mathrm{N}_{\mathrm{a}}\right)$ can be obtained using known relations [9]. The results are summarized in Table 2. It is also observed that the depletion voltage remains almost unchanged after irradiation. This occurs as 


\begin{tabular}{|c|c|c|c|}
\hline Absorbed dose $[\mathrm{kGy}]$ & $\mathrm{V}_{\mathrm{d}}[\mathrm{V}]$ & $\mathrm{C}_{\mathrm{d}}[\mathrm{pF}]$ & $\mathrm{N}_{\mathrm{a}}\left[\mathrm{cm}^{-3}\right]$ \\
\hline 0 & -195.50 & 3.53 & $7.129 \times 10^{12}$ \\
\hline 5 & -223.79 & 3.38 & $7.104 \times 10^{12}$ \\
\hline 10 & -200.05 & 3.43 & $6.723 \times 10^{12}$ \\
\hline 15 & -191.37 & 3.54 & $6.935 \times 10^{12}$ \\
\hline 25 & -193.50 & 3.43 & $6.886 \times 10^{12}$ \\
\hline 30 & -198.12 & 3.43 & $7.003 \times 10^{12}$ \\
\hline
\end{tabular}

Table 2: Various features of a square diode with area $=2.5 \mathrm{~mm} \times 2.5 \mathrm{~mm}$ before and after irradiation; measurement frequency $=100 \mathrm{kHz}$. (Derived from Chapter 4 of [9].)

a result of the oxygen enrichment of silicon which is known to improve the radiation hardness properties of silicon detectors [10].

The IV results were scaled to $20^{\circ} \mathrm{C}$ [11] and the increase of the diode leakage current (absolute value) with the irradiation dose is presented in Fig. 5, left. The increase in $\Delta \mathrm{I}$ with the increase of the absorbed dose can be seen in Fig. 5, right.
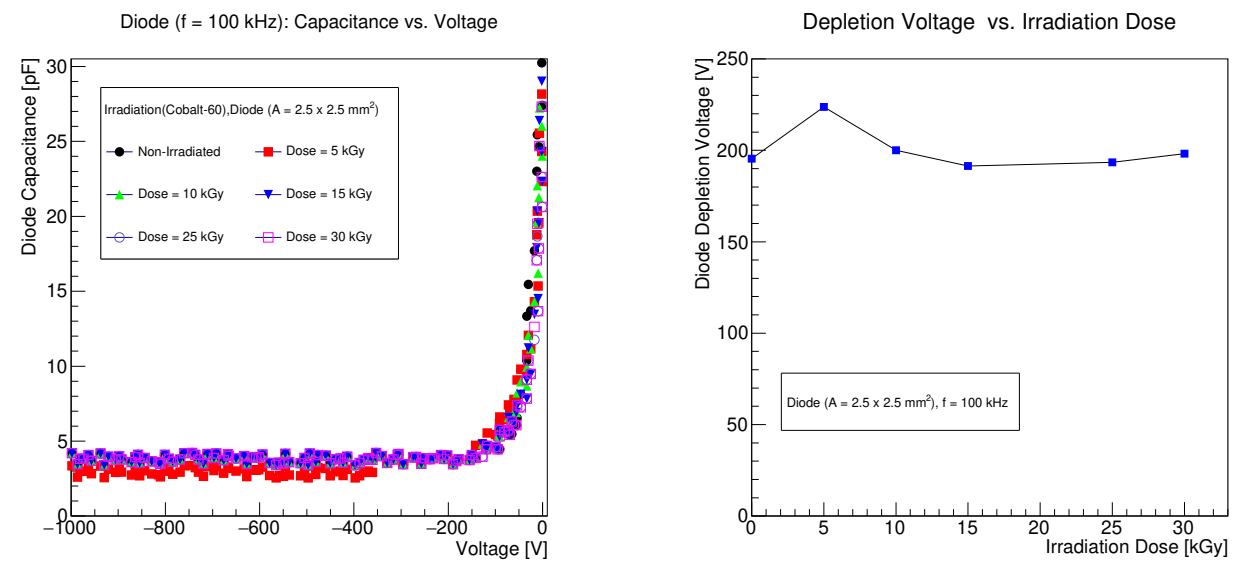

Figure 4: Left: $\mathrm{CV}$ curve for a $2.5 \mathrm{~mm}$ sized diode for various doses; measurement frequency $=100 \mathrm{kHz}$. Right: Depletion voltage as function of the absorbed dose for a $2.5 \mathrm{~mm}$ sized diode; measurement frequency $=100 \mathrm{kHz}$.

\section{Conclusions}

In this work silicon MOS capacitors and n-in-p diodes were irradiated with ${ }^{60} \mathrm{Co}-\gamma$ photons from a $\sim 11 \mathrm{TBq}$ source. The total absorbed dose obtained in several steps was $\sim 30 \mathrm{kGy}$. The level of the radiation-induced charge in the test structures was determined from the shift of the flat band voltage in the MOS after irradiation. In the diodes it was observed that even for high doses the leakage current is in the order of 10-100 nA, while the depletion voltage remains almost unchanged with the increasing dose. This is an indication that the material and the devices are suitable for high-luminosity applications. To understand better the behavior of the material and the 

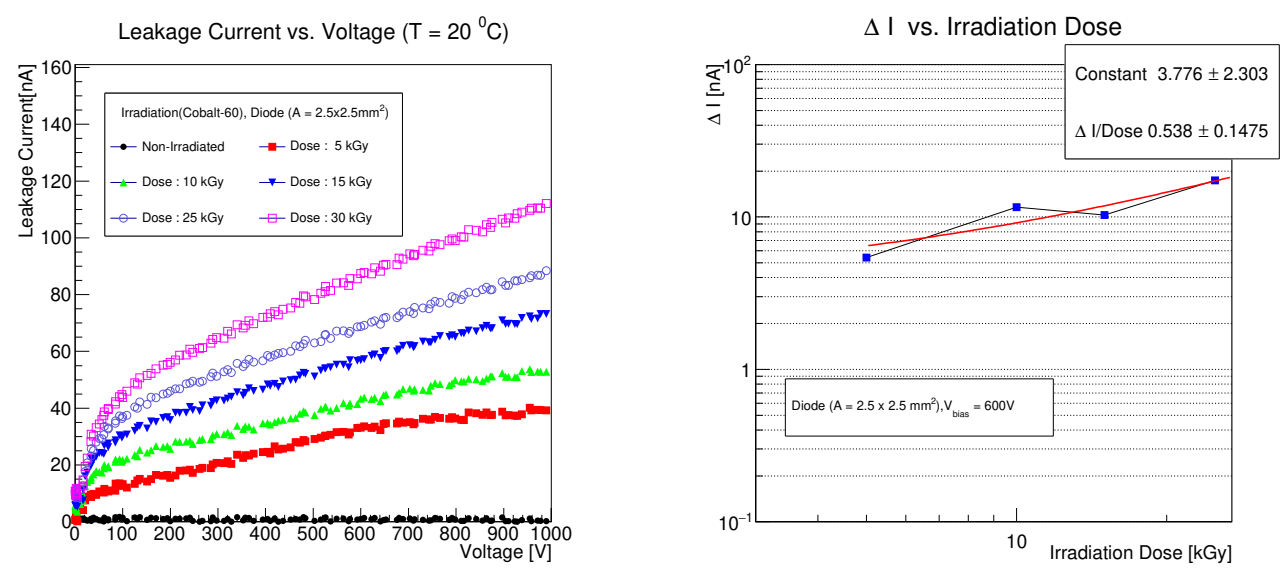

Figure 5: Left: IV curves for a $2.5 \mathrm{~mm}$ sized diode for various doses. Right: $\Delta \mathrm{I}$-dose curve at $\mathrm{V}=-600 \mathrm{~V}$ for a $2.5 \mathrm{~mm}$ sized diode.

devices, the irradiation campaign is still ongoing with the intention of reaching a three times higher absorbed dose compared to this study.

\section{Acknowledgments}

For this work the authors acknowledge the support provided by the General Secretariat of Research and Technology, GSRT (Greece). In addition, Patrick Asenov and Panagiotis Assiouras would like to acknowledge the support by the Hellenic Foundation for Research and Innovation, HFRI (Greece).

\section{References}

[1] G. Apollinari et al., High-Luminosity Large Hadron Collider (HL-LHC): Technical Design Report V. 0.1, CERN Yellow Rep. Monogr. 4 (2017) 1-516 [CERN-2017-007-M].

[2] Georg Steinbrück, The Upgrade of the CMS Outer Tracker, 2014 IEEE Nuclear Science Symposium and Medical Imaging Conference (NSS/MIC) (2014) p. 1-5.

[3] ATLAS Collaboration, ATLAS Phase-II Upgrade Scoping Document, Tech. Rep LHCC-G-166 [CERN-LHCC-2015-020].

[4] H. Simon, "Chapter 1: The Mystique of the Hidden Champions". Hidden Champions of the Twenty-First Century: The Success Strategies of Unknown World Market Leaders, London: Springer Science+Business Media, p.4 ISBN 978-0-387-98147-5.

[5] K. Tsien, R. Robbins, A comparison of a cobalt-60 teletherapy unit and a 2-MEV Van de Graff $x$-ray generator on the basis of physical measurements, Radiology 1958 Apr; 70(4): 486-500; discussion $501-2$.

[6] L. Burigo, S. Greilich, Impact of new ICRU 90 key data on stopping-power ratios and beam quality correction factors for carbon ion beams, Phys Med Biol., 2019 Sep 23; 64(19) :195005.

[7] C.H. Page, P. Vigoureux, eds., The International Bureau of Weights and Measures 1875-1975: NBS Special Publication, 420, Washington, D.C.: National Bureau of Standards pp. 26-27. 
[8] Total dose steady-state irradiation test method, ESCC Basic Specification No. 22900.

[9] Bart V. Van Zeghbroeck, Principles Of Semiconductor Devices And Heterojunctions, Prentice Hall, December 1st 2009.

[10] E. Fretwurst et al., Bulk damage effects in standard and oxygen-enriched silicon detectors induced by 60Co-gamma radiation, Nuclear Instruments and Methods in Physics Research Section A:

Accelerators, Spectrometers, Detectors and Associated Equipment Volume 514, Issues 1-3, 21 November 2003, Pages 1-8.

[11] A. Chilingarov, Generation current temperature scaling, RD50 and EP Technical Note [RD50 TN 2011-01]. 